Diabetologe 2015 · 11:211-218

DOI 10.1007/s11428-015-1335-8

Online publiziert: 29. April 2015

(c) The Author(s) 2015. This article is published

with open access at link.springer.com

B. Kulzer ${ }^{1} \cdot$ B. Lüthgens ${ }^{2} \cdot$ R. Landgraf ${ }^{3} \cdot$ N. Hermanns ${ }^{1}$

${ }^{1}$ Forschungsinstitut der Diabetes-Akademie Bad Mergentheim (FIDAM),

Diabetes Zentrum Mergentheim, Bad Mergentheim

${ }^{2}$ Novo Nordisk Pharma GmbH, Mainz

${ }^{3}$ Deutsche Diabetes-Stiftung, München

\title{
Diabetesbezogene Belastungen, Wohlbefinden und Einstellung von Menschen mit Diabetes
}

\section{Deutsche Ergebnisse der DAWN2 ${ }^{\mathrm{TM}}$-Studie}

Diabetes ist eine chronische Erkrankung, die ein kontinuierliches aktives Selbstmanagement des Patienten erfordert und mit zahlreichen psychosozialen Belastungen verbunden ist [1,2,3]. Bereits im Jahr 2001 konnte die erste globale DAWN („Diabetes Attitudes, Wishes and Needs")-Studie zur Erfassung psychosozialer Bedürfnisse von Menschen mit Diabetes zeigen, dass nach Einschätzung sowohl der Patienten [4] als auch der Behandler [5, 6, 7] eine Reihe psychosozialer Probleme im Zusammenhang mit der Erkrankung besteht. Diese stellen eine wichtige Barriere in Hinblick auf das Erreichen der Ziele der Diabetestherapie dar. Dies gilt sowohl für die Umsetzung der Therapie, das aktive Selbstmanagement der Patienten, die Adhärenz als auch die Therapieergebnisse. Zudem wies ein hoher Anteil von Menschen mit Diabetes eine eingeschränkte Lebensqualität, hohe Belastungen durch diabetesspezifische Stressoren und ein geringes Wohlbefinden auf $[4,8]$. Trotzdem wurden psychologische Aspekte im Rahmen der Diabetestherapie in den meisten Ländern nicht ausreichend berücksichtigt und psychosoziale Angebote waren eher die Ausnahme [7, 8].

Als Konsequenz dieser Studienergebnisse wurde in dem „DAWN-Call-to-Action-Aufruf “ gefordert, dass nachhaltige Anstrengungen notwendig sind, um Menschen mit Diabetes eine individualisierte, patientenzentrierte Therapie $\mathrm{zu}$ ermöglichen und ihnen mehr Unterstüt- zung beim täglichen Selbstmanagement und bei Problemen im Zusammenhang mit der Erkrankung zu geben [9].

\section{》) Die DAWN2 ${ }^{\text {TM}}$-Studie untersucht die psychosoziale Situation von Menschen mit Diabetes}

Trotz zahlreicher Fortschritte seit der ersten DAWN-Studie gibt es noch immer Wissensdefizite bezüglich der Bedürfnisse, Einstellungen und Wünsche von Menschen mit Diabetes, die zudem in der Behandlung des Diabetes zu wenig berücksichtigt werden [10, 11, 12, 13]. Dies war der Grund für die Durchführung der zweiten globalen Studie zu psychosozialen Belastungen und Barrieren der Diabetestherapie: DAWN2 ${ }^{\text {ix }}$ [14]. In dieser Studie wurden psychosoziale Belastungen und Barrieren der Diabetestherapie im Sinne einer 360-Grad-Betrachtung mit teilweise identischen Fragen aus der Perspektive von Menschen mit Diabetes [15], von Angehörigen [16] und von Behandlern [17] betrachtet. Ziel der DAWN2 ${ }^{\text {TM }}$-Studie ist es, mehr Wissen über die psychosoziale Situation, die Bedürfnisse und Probleme von Menschen mit Diabetes, deren Angehörigen als auch der Behandler und Patientenorganisatoren sowie Entscheidungsträger zu erlangen.

Die DAWN2 $2^{\text {is }}$-Studie ist eine globale, partnerschaftliche Initiative verschie- dener internationaler und nationaler Organisationen (International Diabetes Federation [IDF], International Alliance of Patients Organisations [IAPO], Steno Diabetes Center, Dänemark, Firma Novo Nordisk). Die Studie steht im Einklang mit dem „Frameworks for people-centred and innovative chronic illness care" der World Health Organization (WHO) und der „Declaration for patient-centred health care " der IAPO. Die DAWN2 ${ }^{\text {TN }}$ Studie wurde in 17 Ländern auf 4 Kontinenten (Algerien, China, Deutschland, Dänemark, Frankreich, Großbritannien, Niederlande, Indien, Italien, Japan, Kanada, Mexiko, Polen, Russland, Spanien, Türkei und USA) durchgeführt. Insgesamt wurden 15438 Personen befragt. Neben den Menschen mit Diabetes $(n=8596)$ waren dies auch Angehörige von Menschen mit Diabetes $(n=2057)$ und Behandler $(n=4785)$ [14]. In diesem Artikel werden die Ergebnisse der deutschen Stichprobe der DAWN2 $2^{\text {mm }}$-Studie zur psychosozialen Situation von Menschen mit Diabetes sowohl im Vergleich zu Menschen ohne Diabetes (Allgemeinbevölkerung) als auch im Vergleich zu den globalen Daten, welche im Jahr 2013 publiziert wurden [15], berichtet.

\section{Methodik}

Die DAWN2 $2^{\text {mi }}$-Studie wurde von März 2012 bis September 2012 durchgeführt. Die Rekrutierung der deutschen 
Tab. 1 Patientencharakteristik der deutschen Stichprobe von DAWN2 ${ }^{\text {TM }}$

Menschen mit Typ-1- Menschen mit Typ-2-Diabetes P-Wert ${ }^{\mathrm{a}}$

Diabetes $(n=80)$

$(n=422)$

\begin{tabular}{llll}
\hline Alter (MW \pm SD) in Jahren & $39,6 \pm 10,3$ & $57,4 \pm 10,0$ & $<0,001$ \\
\hline Weibliches Geschlecht & $44,4 \%$ & $45,0 \%$ & 0,882 \\
\hline BMI (MW \pm SD) in kg/m ${ }^{2}$ & $24,1 \pm 4,3$ & $29,2 \pm 6,0$ & $<0,001$ \\
\hline $\begin{array}{l}\text { Diabetesdauer (MW } \pm \text { SD) } \\
\text { in Jahren }\end{array}$ & $20,5 \pm 11,9$ & $10,2 \pm 7,9$ & $<0,001$ \\
\hline
\end{tabular}

${ }_{\mathrm{a}}^{\mathrm{p}}$-Werte beziehen sich bei intervallskalierten Daten auf die Ergebnisse eines T-Tests und bei kategorialen Daten für die Ergebnisse eines $x^{2}$-Tests.

Studienteilnehmer im Alter von mindestens 18 Jahren erfolgte telefonisch und online, um die Studienpopulation so repräsentativ wie möglich zu halten und ein potenzielles Bias durch eine Rekrutierung ausschließlich über das Internet $\mathrm{zu}$ vermeiden [14]. Um an der Studie teilzunehmen, mussten Menschen mit Diabetes folgende Einschlusskriterien erfüllen:

- Personen mit Typ-1-Diabetes

- Alter bei Diagnose: <30 Jahre,

- unmittelbare Behandlung mit Insulin,

- aktuelle Insulintherapie,

- Diabetesdauer: mindestens 12 Monaten.

- Personen mit Typ-2-Diabetes

- Alter bei Diagnose: $\geq 30$ Jahre,

- keine Insulinbehandlung nach der Diagnose,

- Diabetesdauer: mindestens 12 Monate.

Patienten unter 18 Jahre und Patientinnen mit Gestationsdiabetes wurden ausgeschlossen. Die deutsche Stichprobe der DAWN2 $2^{\text {is }}$-Studie umfasst 502 Erwachsene mit Diabetes, darunter 80 Menschen mit Typ-1-Diabetes und 422 Menschen mit Typ-2-Diabetes. Die Studienteilnehmer wurden anhand validierter Fragebögen zu diabetesbezogenen Belastungen (PAID-5) [18], ihrem psychischen Wohlbefinden (WHO-5) [19], der Lebensqualität (EQ-5D VAS, Health-Utility-Index) [20], den Auswirkungen des Diabetes auf verschiedene Lebensbereiche (DIDP) [14] und zu Selbstbehandlungsaktivitäten (SDSCA-6) befragt [21]. Außerdem wurden Daten mit dem Patient-Assessment-of-Chronic-Illness-Care-DAWN-Short-Form-Fragebogen (PACIC-DSF) [14] und einer modifizierten Fassung der DiabetesempowermentSkala erhoben [14, 22]. Des Weiteren er- hielten die Teilnehmer Fragen zum Thema soziale Diskriminierung und zu einer Reihe anderer Aspekte im Zusammenhang mit ihrer Diabetestherapie. Von einer Diskriminierung wurde dann ausgegangen, wenn die Befragten der Aussage, dass „sie wegen ihres Diabetes diskriminiert wurden“ im „wesentlichen“ oder „Voll und ganz zustimmten“. Die Methodik der globalen DAWN2 $2^{\text {TM }}$-Studie wurde bereits 2013 publiziert [14].

Alle Teilnehmer erklärten ihre Einwilligung zur Studienteilnahme. In Deutschland wurde diese Studie durch die Ethikkommission der Landesärztekammer Baden Württemberg begutachtet und genehmigt (F-2012-012). Die telefonische Befragung erfolgte in Deutschland durch Harris Interactive (Hamburg). Um eine möglichst hohe Repräsentativität der Befragung zu gewährleisten, wurden die Antworten der untersuchten Stichprobe entsprechend des Alters, Geschlechts und der Schulbildung der deutschen Allgemeinbevölkerung (basierend auf den Statistiken des Statistischen Bundesamtes) gewichtet.

\section{Ergebnisse}

Die globale Stichprobe umfasst insgesamt 8596 Menschen mit Diabetes, die deutsche Stichprobe 502 Personen. Von den 422 Menschen mit Typ-2-Diabetes behandeln 100 ihre Erkrankung ohne Medikamente, 172 mit oralen Antidiabetika und 150 mit Insulin. Die zentralen Charakteristika der deutschen Stichprobe sind in - Tab. 1 aufgeführt. Es zeigen sich im Wesentlichen zu erwartende Unterschiede zwischen Menschen mit Typ-1- und Typ-2-Diabetes in Hinblick auf Alter, Diabetesdauer und Körpergewicht.

\section{Lebensqualität und Wohlbefinden}

Die gesundheitsbezogene Lebensqualität wurde mit dem EQ-5D VAS erhoben, wobei ein Wert von 100 eine sehr gute und ein Wert von 0 eine sehr schlechte Lebensqualität anzeigt. In der deutschen Stichprobe berichten Menschen mit Diabetes im Vergleich zur Allgemeinbevölkerung über eine deutlich reduzierte Lebensqualität; im Vergleich zur globalen Stichprobe zeigen sich keine Unterschiede. Menschen mit Typ-2-Diabetes weisen eine signifikant geringere Lebensqualität auf als Menschen mit Typ-1-Diabetes (- Abb. 1).

\section{\) Psychisches Wohlbefinden ist bei Menschen mit Diabetes im Vergleich zur Allgemein- bevölkerung reduziert}

Ein ähnliches Bild zeigt sich beim psychischen Wohlbefinden, gemessen mit dem WHO-5. Auf einer Skala von 0 bis 100 (maximales Wohlbefinden=100) erreicht die deutsche Allgemeinbevölkerung einen Wert von 70,3. Das Auftreten einer Diabeteserkankung reduziert diesen Wert sehr deutlich auf 57,2. Es gibt keinen signifikanten Unterschied zwischen der deutschen und globalen Stichprobe, ebenfalls nicht zwischen Menschen mit einem Typ-1- oder Typ-2-Diabetes. Ein WHO-5-Score von $\leq 28$ ist mit einer hohen Wahrscheinlichkeit mit dem Auftreten einer Depression assoziiert. Bei etwa jedem 7. Menschen mit Diabetes liegen solche Werte vor (13,6\% für die deutsche bzw. 14,8\% für die globale Stichprobe). Die Wahrscheinlichkeit für eine Depression liegt bei Menschen mit Diabetes damit deutlich höher als in der Allgemeinbevölkerung, bei der nur 5,9\% einen WHO-5Score von $\leq 28$ aufweisen (• Abb. 1).

\section{Diabetesbezogene Belastungen}

Etwa jeder 4. Betroffene der deutschen Stichprobe zeigt eine erhöhte diabetesbezogene Belastung (PAID-5), allerdings signifikant niedriger als in der globalen Stichprobe (• Tab. 2). Ein Blick auf die verschiedenen Lebensbereiche zeigt, dass die Erkrankung für einen nicht ge- 
ringen Anteil von Menschen mit Diabetes mit Belastungen in verschiedenen Lebensbereichen verbunden ist. Am meisten fühlten sich die Studienteilnehmer durch körperliche oder psychische Einbußen aufgrund des Diabetes belastet. Etwa ein Drittel gibt an, aufgrund des Diabetes in den Freizeitaktivitäten beeinträchtigt zu sein. Am geringsten ausgeprägt waren Belastungen im Verhältnis zu Familie, Freunden und Kollegen sowie bei der Arbeit und/oder im Studium. Der deutlichste Unterschied zwischen der globalen und deutschen Stichprobe betrifft finanzielle Belastungen durch den Diabetes (• Abb. 2).

\section{Empowerment}

Das Ausmaß von Empowerment, gemessen mit der DES-Skala (Skala 0-100), ist bei Menschen mit Typ-1-Diabetes (42,9\%) sehr viel höher ausgeprägt als bei Menschen mit Typ-2-Diabetes (26,5\%). Bedingt durch den niedrigen Wert bei Typ2-Diabetes unterscheidet sich hier die deutsche Stichprobe signifikant von der globalen (• Tab. 2).

\section{Selbstbehandlungsverhalten}

Die Auswertung des SDSCA-6 ( $0=$ Umsetzung an keinem Tag der Woche, $7=$ Umsetzung an jedem Tag der Woche) ergab sowohl bei der globalen, als auch der deutschen Stichprobe Hinweise darauf, dass die Adhärenz bezüglich der Umsetzung der Therapie durchaus verbesserungswürdig ist. Die Ergebnisse der deutschen Stichprobe lagen - bis auf den Bereich Ernährung - im Schnitt über den globalen Werten. Die besten Adhärenzwerte wurden in den Bereichen Medikation, Ernährung und Bewegung erreicht (- Tab. 2).

\section{Behandlungszufriedenheit}

Mit der Diabetesbehandlung waren in Deutschland die Befragten (PACIC) sehr viel zufriedener als im globalen Vergleich. In der deutschen Stichprobe äußerten sich Menschen mit Typ-2-Diabetes zufriedener als Menschen mit Typ-1-Diabetes (• Tab. 2).

Diabetologe 2015 · 11:211-218 DOI 10.1007/s11428-015-1335-8

(c) The Author(s) 2015. This article is published with open access at link.springer.com

\section{B. Kulzer $\cdot$ B. Lüthgens $\cdot$ R. Landgraf $\cdot N$. Hermanns \\ Diabetesbezogene Belastungen, Wohlbefinden und Einstellung von Menschen mit Diabetes. Deutsche Ergebnisse der DAWN2 ${ }^{\text {TM }}$-Studie}

\section{Zusammenfassung}

Hintergrund. Ziel der DAWN2 ${ }^{\mathrm{T}}$-Studie ist ein besseres Verständnis von Bedürfnissen und Problemen von Menschen mit Diabetes, deren Angehörigen, Behandlern und Patientenorganisationen bzw. Entscheidungsträgern. Es werden die Ergebnisse der deutschen Stichprobe berichtet und mit den internationalen Ergebnissen verglichen. Methodik. An der DAWN2 ${ }^{\mathrm{TM}}$-Studie nahmen 8596 Menschen mit Diabetes aus 17 Ländern in 4 Kontinenten teil. In jedem Land wurden ca. 500 Menschen mit Diabetes befragt. Die deutsche Teilstichprobe umfasst 502 Personen (80 Menschen mit Typ-1-Diabetes und 422 Menschen mit Typ-2-Diabetes). Es wurden standardisierte Fragebögen zur Erfassung der Lebensqualität, der diabetesbezogenen Belastungen, des Selbstbehandlungsverhaltens, dem Diabetes-Empowerment und der Behandlungszufriedenheit eingesetzt und nach sozialer Diskriminierung gefragt.

Ergebnisse. Diabetespatienten in Deutschland haben im Vergleich zur Allgemeinbevölkerung eine reduzierte Lebensqualität und

\section{Diabetes-related stress, well-being, and attitudes of people with diabetes. German results of the DAWN2 ${ }^{\mathrm{Tm}}$ study}

\section{Abstract}

Background. The DAWN2 ${ }^{\text {TM }}$ study aims to improve the understanding of problems and unmet needs of people with diabetes and their family members, as well as healthcare professionals, patient organizations, and key stakeholders in diabetes. This article reports the results for the German study population and compares them to the global results. Methods. A total of 8596 people with diabetes participated in the global DAWN2 ${ }^{\text {TM }}$ study which was conducted in 17 countries representing 4 continents. Per country about 500 people with diabetes participated in the trial. The German study population comprised 502 people with diabetes ( 80 people with type 1 diabetes, 422 people with type 2 diabetes). Validated questionnaires for standardized measurement of quality of life, diabetes-related distress, diabetes self-care activities, empowerment, and healthcare provision were used. Additional items, e.g. social discrimination, were included.

Results. Compared to the general population in Germany, people with diabetes re- ein geringeres Wohlbefinden. Trotz dieses negativen Einflusses der Erkrankung auf die Lebensqualität werden psychosoziale Aspekte in der Diabetesversorgung relativ selten thematisiert. Im globalen Vergleich zeigen sich eine hohe Behandlungszufriedenheit und eine hohe Schulungsrate. Das Ausmaß an sozialer Diskriminierung ist bemerkenswert hoch, jedoch niedriger als im internationalen Vergleich.

Schlussfolgerung. Die DAWN2 ${ }^{\text {TM }}$-Studie zeigt, dass Diabetes mit einer Reihe psychosozialer Belastungen verbunden ist. Es gibt erhebliche Defizite in der psychosozialen Versorgung von Menschen mit Diabetes, die es im Interesse der Betroffenen zu verbessern gilt.

Register klinischer Studien. „Universal tri-

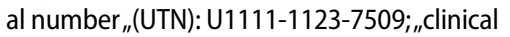
trials gov identifier" (NCT): NCT01507116.

\section{Schlüsselwörter}

Lebensqualität · Depression · Psychosoziale

Belastungen · Diabetesschulung ·

Diskriminierung ported a reduced quality of life and emotional well-being. Dispite this negative impact of diabetes on the quality of life, psychosocial aspects are rarely integrated in routine diabetes care. Nevertheless, compared to global results, the German results show a high satisfaction with diabetes care in general and a high rate of diabetes education. The extent of social discrimination is notably high, but lower than in the global comparison.

Conclusion. The DAWN2 ${ }^{\mathrm{TM}}$ study reveals that diabetes is associated with significant psychosocial burden. There is a need for improvement in psychosocial care for people with diabetes.

Clinical trial registration. Universal Trial Number (UTN): U1111-1123-7509; Clinical Trials gov Identifier (NCT): NCT01507116.

Keywords

Quality of life - Depression · Psychosocial distress - Diabetes education - Discrimination 


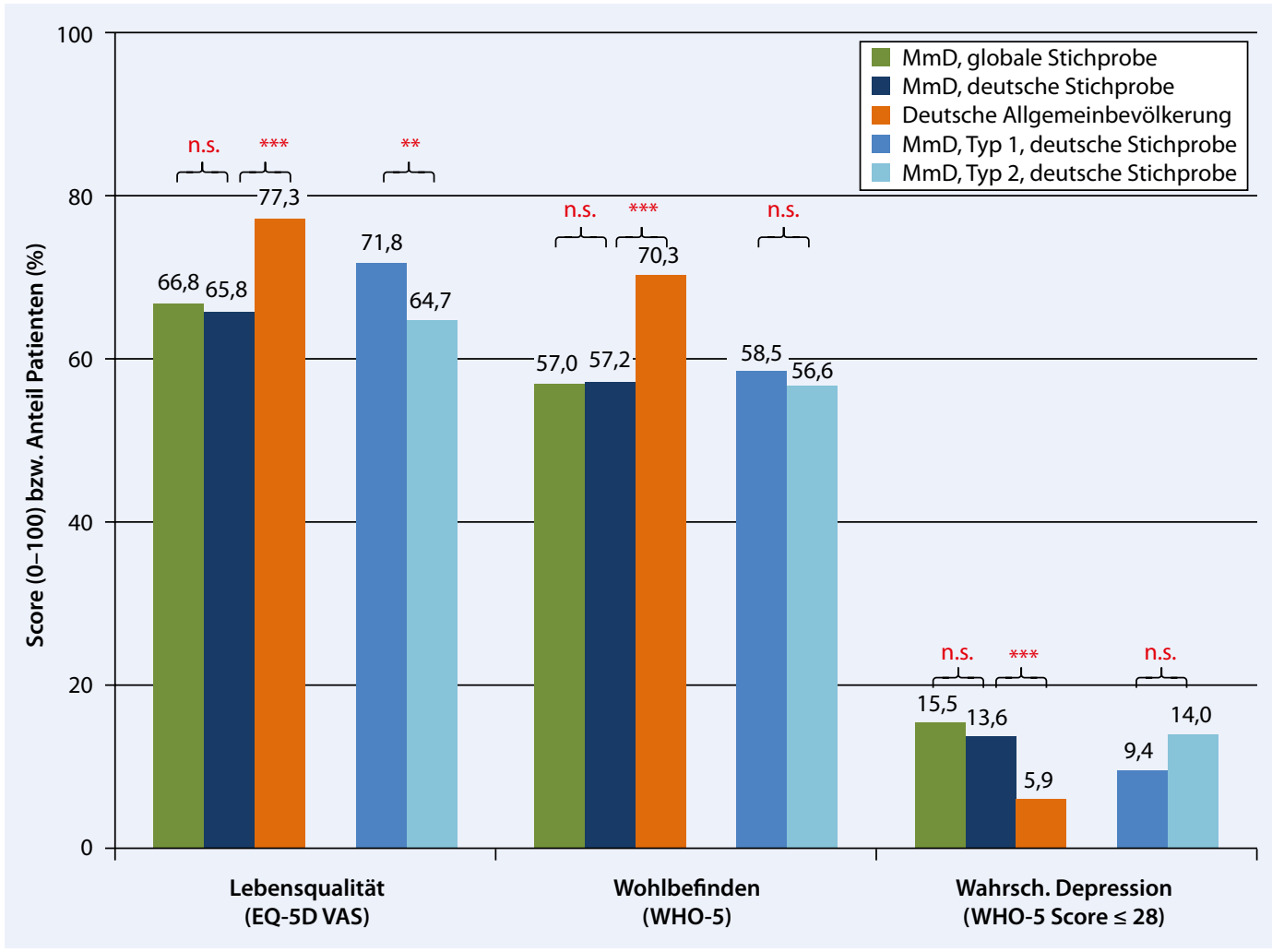

Abb. $1<$ Gesundheitsbezogene Lebensqualität (EQ-5D VAS), psychisches Wohlbefindens (WHO-5) und wahrscheinliche Depression (WHO-5-Score $\leq 28$ ). Vergleich der deutschen DAWN2 ${ }^{\text {TM }}$-Stichprobe mit der globalen DAWN2 ${ }^{\mathrm{TM}}$-Stichprobe und mit der deutschen Normstichprobe (Allgemeinbevölkerung $[19,20])$ sowie getrennt nach Typ-1Diabetes vs. Typ-2-Diabetes. (n.s. nicht signifikant, $M m D$ Menschen mit Diabetes, ${ }^{*} p<0,05 ;{ }^{* *} p<0,01$; $* * * p<0,001$ )

\section{Diagnostische Maßnahmen}

Auf die Frage, welche diagnostischen Maßnahmen im letzten Jahr durchgeführt wurden, ergibt sich für die deutsche wie auch die globale Stichprobe ein ähnliches Bild. Die meisten Studienteilnehmer gaben an, dass klassische diagnostische Maßnahmen durchgeführt wurden wie die Bestimmung des $\mathrm{HbA}_{1 \mathrm{c}}$-Wertes, des Blutdrucks, der Blutfettwerte und eine Fußuntersuchung (Letzteres allerdings auch in Deutschland nur bei 65\% der Personen). Nur bei weniger als der Hälfte aller Patienten wurde vom Arzt bzw. dem Diabetesteam nach Ernährungs- (41,5\%) bzw. Bewegungsgewohnheiten (46,0\%) gefragt. Dagegen wurde nur bei ca. jedem 3. Patient nach einer möglichen Depression gefragt. Noch seltener wurde der Einfluss des Diabetes auf den Alltag bzw. die Lebensqualität thematisiert.

\section{》) Die Bestimmung von Langzeitzuckerwerten $\left(\mathrm{HbA}_{1 c}\right)$ erfolgt häufiger als im globalen Durchschnitt}

Signifikant häufiger als im globalen Durchschnitt wurden in Deutschland der $\mathrm{HbA}_{1 \mathrm{c}}$-Wert bestimmt und die Füße kontrolliert. Bei der Frage nach möglichen psychosoziale Belastungen im Zusammenhang mit der Diabeteserkrankung gab es keinen signifikanten Unterschied zu den globalen Ergebnissen (• Abb. 3).

\section{Diabetesschulung}

Bei der Frage nach dem Besuch einer strukturierten Diabetesschulung oder einer Einzelschulung nimmt Deutschland im globalen Vergleich einen Spitzenplatz ein: Knapp 80\% der Menschen mit Diabetes in Deutschland gaben an, schon einmal geschult worden zu sein, während dies in der globalen Stichprobe nur bei knapp $60 \%$ der Fall ist. Trotz einer höheren Schulungsgrate bei Typ-1-Diabetes gibt es keinen signifikanten Unterschied zwischen Typ-1- und Typ-2-Diabetes (• Tab. 2).

\section{Soziale Diskriminierung}

In der globalen Stichprobe berichtete etwa jeder 6. Mensch mit Diabetes, sich wegen der Diabeteserkrankung stark oder sehr stark diskriminiert zu fühlen. Dieser Anteil war in der deutschen Stichprobe mit insgesamt $10,6 \%$ signifikant niedriger. Menschen mit einem Typ-1-Diabetes haben hierbei signifikant häufiger das Gefühl, wegen der Diabeteserkankung diskriminiert zu werden: Dies betraf jeden 5. Menschen mit Typ-1-Diabetes (• Abb. 4).

\section{Diskussion}

Der Erhalt einer optimalen Lebensqualität ist ein wesentliches Ziel der Diabetestherapie [12, 13]. Vor diesem Hintergrund ist die in der deutschen Stichprobe beobachtete signifikante Reduktion der gesundheitsbezogenen Lebensqualität von Menschen mit Diabetes im Vergleich zur Allgemeingemeinbevölkerung besonders bedeutsam. Die mit einer Diabeteserkrankung assoziierten Einbußen an gesundheitsbezogener Lebensqualität beträgt etwa 10 Prozentpunkte auf einer Skala von 0 (minimale Lebensqualität) bis 100 (maximale Lebensqualität) und ist klinisch relevant. 
Tab. 2 Ergebnisse der deutschen und globalen Stichprobe der DAWN2 ${ }^{\text {TM }}$-Studie

$\begin{array}{lllll}\begin{array}{l}\text { Menschen mit Typ- } \\ \text { 1-Diabetes }(n=80)\end{array} & \begin{array}{l}\text { Menschen mit Typ-2- } \\ \text { Diabetes }(n=422)\end{array} & \text { P-Wert }^{\text {a }} & \begin{array}{l}\text { Deutsche Stich- } \\ \text { probe }(n=502)\end{array} & \begin{array}{l}\text { Globale } \\ \text { Stichprobe } \\ (n=8596)\end{array}\end{array} \quad$ P-Wert $^{\text {a }}$

\begin{tabular}{|c|c|c|c|c|c|c|}
\hline \multicolumn{7}{|l|}{ Diabetesbezogene Belastungen (PAID-5) } \\
\hline Mittlere diabetesbezogene Belastungen & 33,9 & 24,2 & $<0,01$ & 25,3 & 34,1 & $<0,01$ \\
\hline $\begin{array}{l}\text { Hohe diabetesbezogener Belastung, } \\
\text { PAID-5-Score } \geq 40(\%)\end{array}$ & 43,5 & 24,9 & $<0,01$ & 27,3 & 42,7 & $<0,01$ \\
\hline \multicolumn{7}{|l|}{ Empowerment (DES) } \\
\hline Mittlerer DES-Score & 42,9 & 26,5 & $<0,01$ & 29,4 & 37,6 & $<0,01$ \\
\hline \multicolumn{7}{|c|}{$\begin{array}{l}\text { Selbstbehandlungsverhalten (SDSCA-6) } \\
\text { Wie oft haben Sie in den vergangenen } 7 \text { Tagen... }\end{array}$} \\
\hline $\begin{array}{l}\text {...einen gesunden Ernährungsplan be- } \\
\text { folgt? }\end{array}$ & 4,6 & 4,7 & 0,434 & 4,7 & 4,9 & $<0,01$ \\
\hline ...sich mind. 30 min körperlich betätigt? & 4,5 & 4,4 & 0,594 & 4,4 & 3,4 & $<0,01$ \\
\hline ...Ihren Blutzucker gemessen? & 5,6 & 3,8 & $<0,01$ & 4,0 & 3,6 & $<0,01$ \\
\hline $\begin{array}{l}\text {... Ihren Blutzucker so oft gemessen, wie } \\
\text { es Ihr Arzt empfohlen hat? }\end{array}$ & 5,6 & 4,0 & $<0,01$ & 4,2 & 3,4 & $<0,01$ \\
\hline ... Ihre Füße überprüft? & 3,7 & 3,9 & 0,354 & 4,0 & 3,5 & $<0,01$ \\
\hline $\begin{array}{l}\text {... Ihre Diabetesmedikamente so ein- } \\
\text { genommen, wie mit Ihrem Arzt abge- } \\
\text { sprochen? }\end{array}$ & 5,6 & 6,5 & $<0,01$ & 6,3 & 6,2 & 0,823 \\
\hline \multicolumn{7}{|l|}{ Behandlungszufriedenheit (PACIC) } \\
\hline Mittlerer PACIC-Score & 53,2 & 66,9 & 0,025 & 65,0 & 49,5 & $<0,01$ \\
\hline \multicolumn{7}{|l|}{ Patientenschulung } \\
\hline Teilnahme an Schulung (\%) & 87,6 & 78,5 & 0,077 & 78,7 & 58,8 & $<0,01$ \\
\hline
\end{tabular}

> Eine Diabeteserkankung wirkt sich deutlich negativ auf die gesundheitsbezogene Lebensqualität aus.

Diese Ergebnisse korrespondieren sehr gut mit einer Analyse von Schunk et al. [23], in der die Daten von 5 populationsbasierten epidemiologischen Erhebungen aus Deutschland gepoolt wurden und ebenfalls bei Menschen mit Diabetes eine Reduktion der gesundheitsbezogenen Lebensqualität in einer vergleichbaren Dimension festgestellt wurde. Diese Reduktion ist bei Menschen mit einem Typ-2Diabetes signifikant stärker ausgeprägt. Auch wenn die gesundheitsbezogene Lebensqualität der deutschen Teilstichprobe in etwa dem globalen Durchschnitt entspricht, zeigt die Verminderung der Lebensqualität bei Menschen mit Diabetes in Relation zur deutschen Allgemeinbevölkerung, dass ein zentrales Ziel der Behandlung von Menschen mit Diabetes der Erhalt der Lebensqualität trotz und mit Diabetes - nicht erreicht wurde.

Neben der gesundheitsbezogenen Lebensqualität zeigte sich auch ein im Vergleich zur deutschen Allgemeinbevölke- rung signifikant reduziertes emotionales Wohlbefinden. Bei etwa jedem 7. Menschen mit Diabetes ist das emotionale Wohlbefinden derart beeinträchtigt, dass das Vorliegen einer Depression wahrscheinlich ist. Eine regelmäßige strukturierte Erhebung und Dokumentation des Wohlbefindens, wie es von der International Diabetes Federation (IDF) und der Deutschen Diabetes Gesellschaft (DDG) $[12,13]$ empfohlen wird (siehe auch Gesundheits-Pass Diabetes), erscheint daher sehr angemessen. Auf dem Hintergrund einer deutlichen Einschränkung der gesundheitsbezogenen Lebensqualität und des Wohlbefindens ist die Tatsache, dass nur bei rund einem Drittel aller Betroffenen das psychosoziale Wohlbefinden routinemäßig bei Arztbesuchen thematisiert wird, besonders problematisch.

Zudem empfinden mehr als ein Viertel aller Menschen mit Diabetes in Deutschland eine hohe emotionale Belastung wegen ihres Diabetes. Daher ist es sinnvoll, neben dem allgemeinen Wohlbefinden regelmäßig auch das Vorliegen von diabetesbezogenen Belastungen zu erfragen. Interessanterweise liegen jedoch die diabetesbezogenen Belastungen deutlich unterhalb des internationalen Durchschnitts. Evaluationsstudien zur Diabetesschulung bei Typ-1- [24] und Typ-2-Diabetes $[25,26]$ legen nahe, dass mit einer Teilnahme an einer Diabetesschulung sowohl die Fertigkeiten und Fähigkeiten zur Diabetesbehandlung verbessert, als auch diabetesbezogene Belastungen reduziert werden können. Die im globalen Vergleich hohe Schulungsrate in Deutschland könnte eine wesentliche Ursache für die deutlich niedrigeren diabetesbezogenen Belastungen der deutschen Stichprobe sein. Dennoch erreichen Menschen mit Diabetes im globalen Vergleich einen deutlich geringeren Wert in der DiabetesEmpowerment-Skala. Dies ist besonders bei Menschen mit einem Typ-2-Diabetes der Fall. Dies könnte ein Hinweis darauf sein, dass in Deutschland besonders im Bereich Typ-2-Diabetes eher traditionelle, primär auf eine Wissensvermittlung abzielende Schulungsprogramme noch immer eine hohe Verbreitung haben [27].

Bei der Erhebung des Selbstbehandlungsverhaltens ergab sich eine relativ hohe Therapieadhärenz bezüglich der Me- 


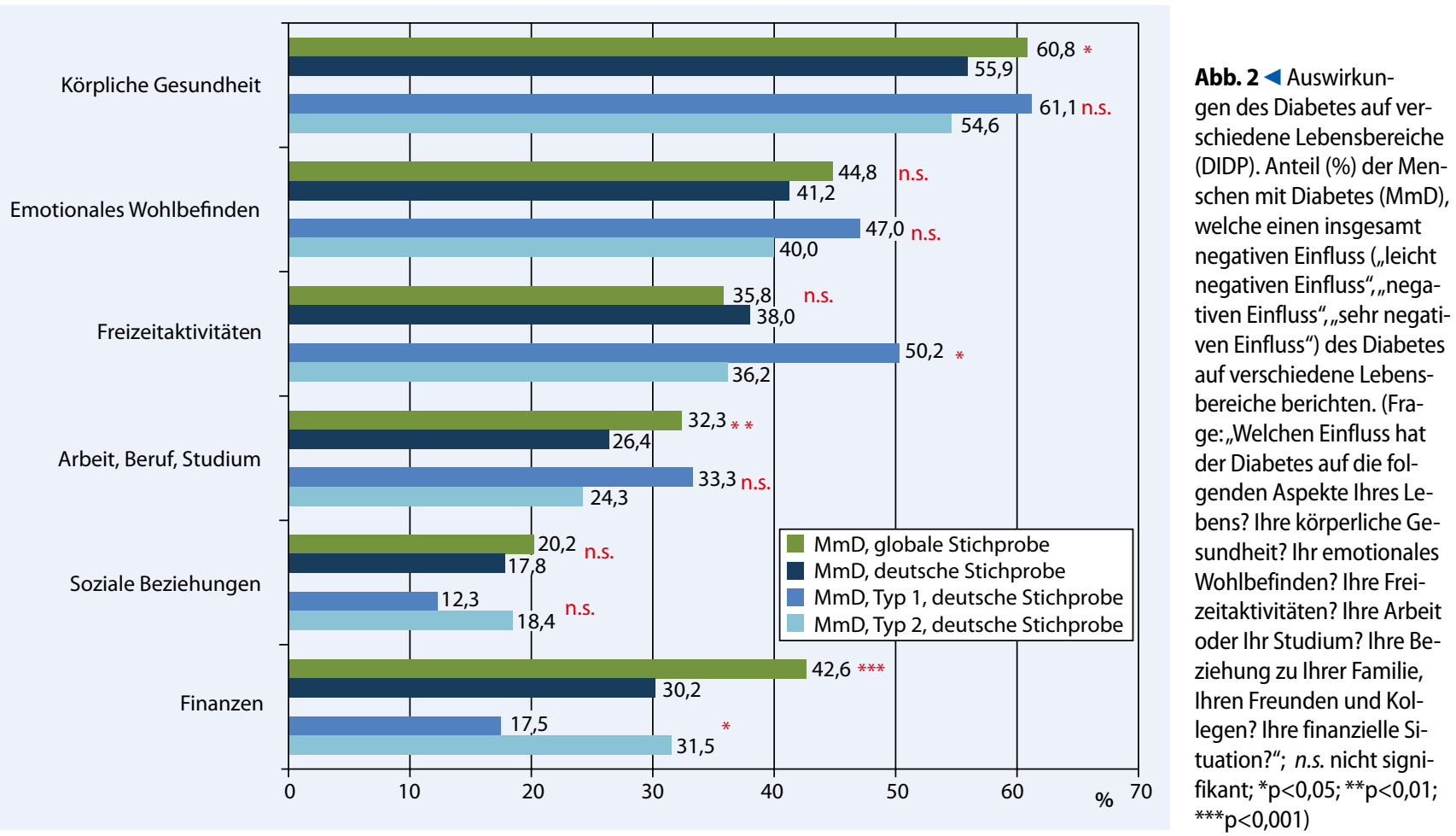

dikamenteneinnahme bzw. Insulininjektionen, während Selbstbehandlungsmaßnahmen, welche mit einer Veränderung des Lebensstils (z. B. Bewegung, Ernährung) einhergehen, eine deutlich geringere Adhärenz aufwiesen. Allerdings war in der deutschen Stichprobe in 4 von 6 befragten Bereichen eine signifikant höhere Therapieadhärenz zu verzeichnen als in der globalen Stichprobe. Auch hierzu könnte der hohe Schulungsstand beigetragen haben $[28,29]$. Dazu passt auch, dass im Vergleich zur globalen Stichprobe die deutschen Studienteilnehmer über eine deutlich höhere Behandlungszufriedenheit berichteten.

Interessant sind die Ergebnisse, nach denen diagnostische Maßnahmen zur Bestimmung des $\mathrm{HbA}_{1 \mathrm{c}}$-Wertes, des Blutdrucks, der Blutfette sehr viel häufiger durchgeführt werden als die Untersuchung der Füße und v. a. das Nachfragen zur Umsetzung von Lebensstilmodifikationen. Besonders selten werden bei Menschen mit einer Diabeteserkrankung psychische Belastungsfaktoren im Zusammenhang mit dem Diabetes und die Auswirkungen des Diabetes auf verschiedene Lebensbereiche thematisiert. Vor dem Hintergrund der geschilderten psychosozialen Belastungen ist dieser Befund besonders problematisch und verbesserungswürdig.

\section{) Soziale Diskriminierung im Zusammenhang mit Diabetes ist ein weltweites Problem}

Bislang gibt es kaum Daten zum Thema soziale Diskriminierung. Es ist ein Verdienst der DAWN2 $2^{\text {in }}$-Studie hierzu erstmals Daten zu liefern. Natürlich basieren diese Daten zur Diskriminierung auf der subjektiven Wahrnehmung der Befragten und ist nicht an objektiven Gegebenheiten validiert. Trotzdem weisen die globalen Ergebnisse darauf hin, dass soziale Diskriminierung im Zusammenhang mit dem Diabetes ein weltweites Problem darstellt. Positiv zu vermerken ist, dass in der deutschen Stichprobe im weltweiten Vergleich eine relativ niedrige Diskriminierungsrate festzustellen ist. Allerdings stimmt bedenklich, dass auch in Deutschland jeder 10. Mensch mit Diabetes angibt, sich aufgrund der Erkrankung diskriminiert zu fühlen, bei Typ-1-Diabetes ist es sogar jede 5. Person. Möglicherweise erleben Menschen mit einem Typ-1-Diabetes Einschränkungen in ihrer Berufswahl, Benachteiligungen im Berufsleben, Probleme im Zusammenhang mit der Fahr- tauglichkeit oder Versicherungen deutlich stärker als Menschen mit einem Typ-2Diabetes. Dies deutet darauf hin, dass ein nicht geringer Prozentsatz von Menschen mit Diabetes sich wegen der Erkrankung benachteiligt fühlt und zukünftig geeignete Anstrengungen unternommen werden sollten, um diesen Zustand zu ändern.

Für die Interpretation der Ergebnisse sollten auch potenzielle Limitationen der Studie berücksichtigt werden. Alle Ergebnisse basieren auf Selbstberichtsverfahren, sodass die bekannten einschränkenden Bedingungen dieser Methode wie Verzerrung von Ergebnissen durch Erinnerungsfehler, subjektive Gewichtung von Fakten, Tendenz zur sozialen Erwünschtheit als potenzielle Fehlerquellen bei der Interpretation der Ergebnisse berücksichtigt werden sollten. Diese Limitation trifft sowohl für die nationale als auch die globale Stichprobe gleichermaßen zu. Obwohl die Gewichtung von Antworten entsprechend zentraler Kenngrößen der Allgemeinbevölkerung ein gängiges Verfahren ist, um die Repräsentativität von Befragungsergebnissen zu erhöhen, kann die Repräsentativität dieser Ergebnisse nicht positiv bewiesen werden. Zudem kann die in Deutschland verwendete Untersuchungsmethode mit Telefoninterviews zu möglichen Verzerrungen des Antwortver- 


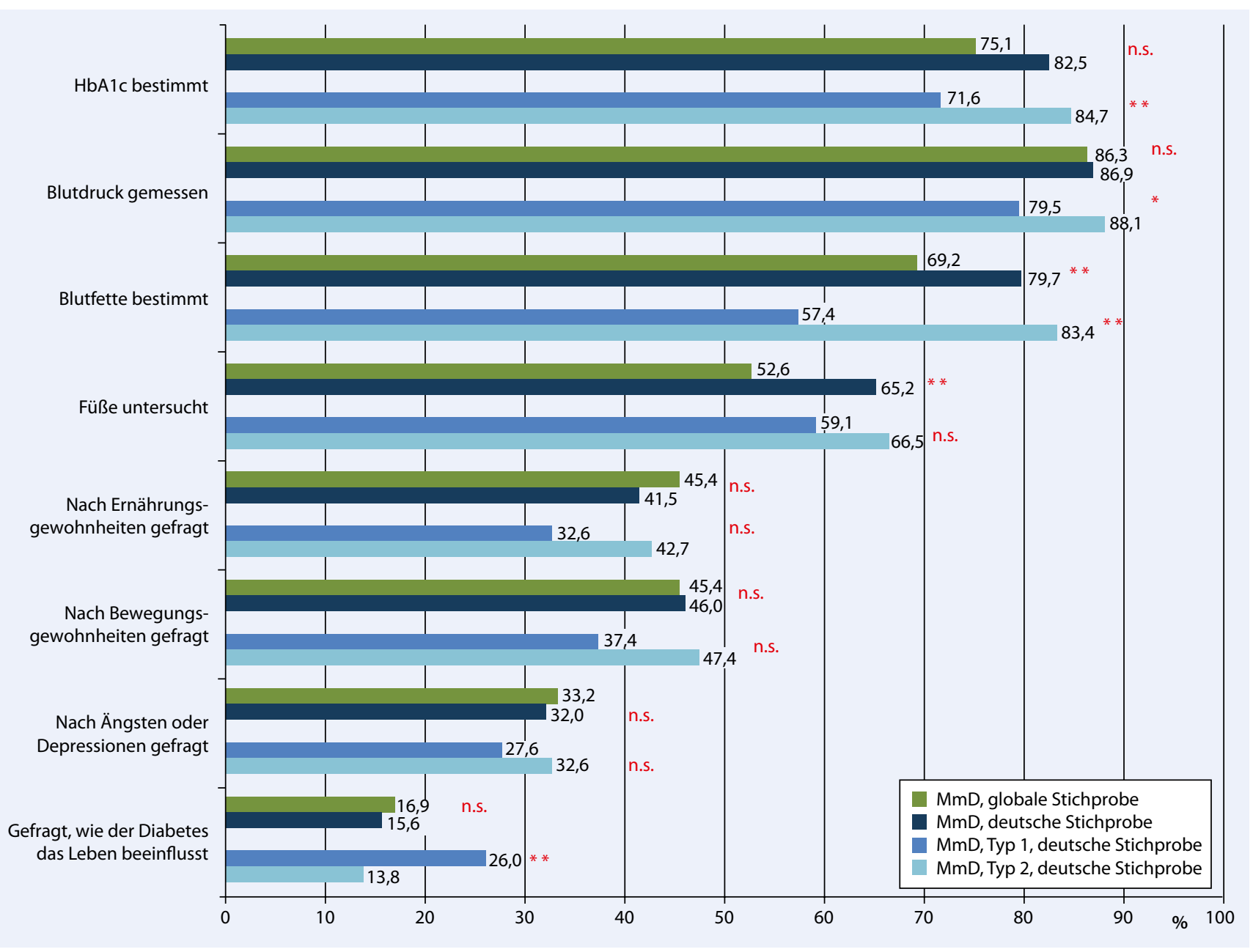

Abb. $3 \Delta$ Durchführung von diagnostischen Maßnahmen. Anteil (\%) der Menschen mit Diabetes (MmD), welche angeben, dass bei ihnen in den letzten 12 Monaten bestimmte diagnostische Maßnahmen durch das Behandlungsteam durchgeführt wurden. (Frage: „Hat in den vergangenen 12 Monaten jemand von ihrem Behandlungsteam bei Ihnen folgende Maßnahmen durchgeführt? Ihren Langzeitblutzucker $\left[\mathrm{HbA}_{1 \mathrm{c}}\right]$ gemessen? Ihren Blutdruck gemessen? Ihre Blutfettwerte gemessen? Ihre Füße untersucht? Sie nach ihren Ernährungsgewohnheiten gefragt? Sie nach ihren Bewegungsgewohnheiten gefragt? Sie gefragt, ob Sie ängstlich oder depressiv sind? Sie gefragt, wie der Diabetes Ihr Leben beeinflusst?, n.s. nicht signifikant; $\left.{ }^{*} \mathrm{p}<0,05 ;{ }^{* *} \mathrm{p}<0,01 ;{ }^{* * *} \mathrm{p}<0,001\right)$

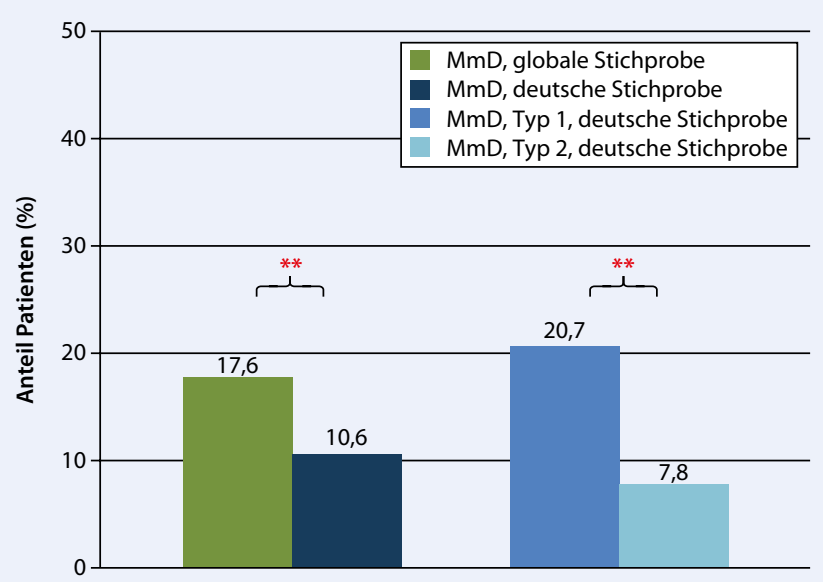

Abb. $4<$ Diskriminierung aufgrund der Diabeteserkrankung. Anteil (\%) der Menschen mit Diabetes (MmD), die angeben, sich aufgrund des Diabetes diskriminiert zu fühlen haltens geführt haben. Bei dem Vergleich mit den globalen Daten muss außerdem berücksichtigt werden, dass in den verschiedenen Ländern unterschiedliche Erhebungsmethoden (z. B. persönliche Befragung, Telefoninterviews, Online-Befragung) mit unterschiedlichem Verzerrungspotenzial eingesetzt wurden. Obwohl in der DAWN2 $2^{\text {mw }}$-Studie überwiegend bereits evaluierte Fragebögen zum Einsatz kamen, wurden auch eigens für diese Studie konstruierte Frageninventars eingesetzt, deren Reliabilität und Validität nicht in allen der 17 beteiligten Ländern überprüft wurde (z. B. Fragen zur Diabetesschulung, soziale Diskriminierung). Trotz dieser Limitationen erbrach- 
te die DAWN2 ${ }^{\text {rm }}$-Studie wichtige und bisher nicht erforschte Erkenntnisse in Hinblick auf psychosoziale Probleme im Umgang mit dem Diabetes in Deutschland und einer größeren multinationalen Stichprobe.

\section{Fazit}

Zusammenfassend belegen die Daten der DAWN2 ${ }^{\text {TM }}$-Studie, dass auch 10 Jahre nach Abschluss der ersten DAWN ${ }^{\mathrm{Tm}}$-Studie, psychosoziale Probleme von Menschen mit einer Diabeteserkrankung noch immer nicht hinreichend Berücksichtigung finden. In Deutschland stellt sich die Situation im Hinblick auf die Schulungsteilnahme, Therapieadhärenz und soziale Diskriminierung zwar etwas günstiger dar als in der globalen Stichprobe, trotzdem gibt es auch hier eine bedeutsame Lücke zwischen den psychosozialen Bedürfnissen von Menschen mit Diabetes und entsprechenden Angeboten in der klinischen Praxis, die es zu schließen gilt.

\section{Korrespondenzadresse}

PD Dr. phil. B. Kulzer

Forschungsinstitut der DiabetesAkademie Bad Mergentheim (FIDAM), Diabetes Zentrum Mergentheim Theodor-Klotzbücher-Str. 12, 97980 Bad Mergentheim

kulzer@diabetes-zentrum.de

\section{Einhaltung ethischer Richtlinien}

Interessenkonflikt. Bernd Kulzer (BK) ist Mitglied des Nationalen DAWN2 ${ }^{\mathrm{TM}}$ Advisory Boards, des Advisory Board der Novo Nordisk Akademie und verantwortlicher Leiter der Deutschen DAWN2 ${ }^{\mathrm{TM}}$-Studie (Lead National Investigator). Für Vortrags- und Beratertätigkeiten erhielt er Honorare von Novo Nordisk. Birgit Lüthgens (BL) ist bei Novo Nordisk Pharma GmbH beschäftigt und Leiterin der Novo Nordisk Akademie. Rüdiger Landgraf (RL) ist Mitglied des Nationalen DAWN2 ${ }^{\mathrm{TM}}$ Advisory Boards und des Advisory Board der Novo Nordisk Akademie. Für Vortrags- und Beratertätigkeiten erhielt er Honorare von Novo Nordisk. Norbert Hermanns (NH) ist Mitglied des Nationalen DAWN2 ${ }^{\mathrm{TM}} \mathrm{Ad}-$ visory Boards und des Internationalen DAWN2 ${ }^{\mathrm{TM}}$ Publication Planning Committee. Für Vortrags- und Beratertätigkeiten erhielt er Honorare von Novo Nordisk.

Dieser Beitrag beinhaltet keine Studien an Menschen oder Tieren.

\section{Open Access}

This article is distributed under the terms of the Creative Commons Attribution 4.0 International License (http://creativecommons.org/licenses/by/4.0/), which permits unrestricted use, distribution, and reproduction in any medium, provided you give appropriate credit to the original author(s) and the source, provide a link to the Creative Commons license, and indicate if changes were made.

\section{Literatur}

1. Polonsky WH, Fisher L, Earles J et al (2005) Assessing psychosocial distress in diabetes: development of the diabetes distress scale. Diabetes Care 28:626-631

2. Kulzer B (2012) Verbesserte Versorgung von Menschen mit Diabetes. Nationaler Diabetesplan. Kompendium Diabetes 7:25-31

3. Kulzer B, Hermanns N (2011) Diabetes als verhaltensmedizinische Erkrankung. In: Häring H-U, Gallwitz B, Müller-Wieland D, Usadel KH, Mehnert $\mathrm{H}$ (Hrsg) Diabetologie in Klinik und Praxis. 6. Aufl., vollst. überarb. erw. ed. Thieme, Stuttgart, S 268282

4. Peyrot M, Rubin RR, Lauritzen T et al (2005) Psychosocial problems and barriers to improved diabetes management: results of the Cross-National Diabetes Attitudes, Wishes and Needs (DAWN) Study. Diabet Med 22:1379-1385

5. Peyrot M, Rubin RR, Siminerio LM (2006) Physician and nurse use of psychosocial strategies in diabetes care: results of the cross-national Diabetes Attitudes, Wishes and Needs (DAWN) study. Diabetes Care 29:1256-1262

6. Peyrot M, Rubin RR, Lauritzen T et al (2006) Patient and provider perceptions of care for diabetes: results of the cross-national DAWN Study. Diabetologia 49:279-288

7. Siminerio $L M$, Funnell MM, Peyrot $M$, Rubin RR (2007) US nurses' perceptions of their role in diabetes care: results of the cross-national Diabetes Attitudes Wishes and Needs (DAWN) study. Diabetes Educ 33:152-162

8. Rubin RR, Peyrot M, Siminerio LM (2006) Health care and patient-reported outcomes: results of the cross-national Diabetes Attitudes, Wishes and Needs (DAWN) study. Diabetes Care 29:1249-1255

9. o A (2004) Conference Report: 2nd International DAWN Summit: 2nd International DAWN Summit: a call - to-action to improve psychosocial care for people with diabetes. Pract Diabetes Int 21:201208. http://www.dawnstudy.com/documents/ DAWN\%20materials/DAWN_Publications/13_call to_action_to_improve.pdf

10. Glasgow RE, Peeples M, Skovlund SE (2008) Where is the patient in diabetes performance measures? The case for including patient-centered and selfmanagement measures. Diabetes Care 31:10461050

11. Snoek FJ, Kersch NY, Eldrup E et al (2011) Monitoring of Individual Needs in Diabetes (MIND): baseline data from the Cross-National Diabetes Attitudes, Wishes, and Needs (DAWN) MIND Study. Diabetes Care 34:601-603

12. Kulzer B, Albus C, Herpertz S et al (2013) Psychosoziales und Diabetes (Teil 1). S2-Leitlinie Psychosoziales und Diabetes - Langfassung. Diabetol Stoffwechs 8:198-242

13. Kulzer B, Albus C, Herpertz S et al (2013) Psychosoziales und Diabetes (Teil 2). S2-Leitlinie Psychosoziales und Diabetes - Langfassung. Diabetol Stoffwechs 8:292-324
14. Peyrot M, Burns KK, Davies M et al (2013) Diabetes Attitudes Wishes and Needs 2 (DAWN2): a multinational, multi-stakeholder study of psychosocial issues in diabetes and person-centred diabetes care. Diabetes Res Clin Pract 99:174-184

15. Nicolucci A, Kovacs Burns K, Holt Rl et al (2013) Diabetes attitudes, wishes and needs second study (DAWN2): cross-national benchmarking of diabetes-related psychosocial outcomes for people with diabetes. Diabet Med 30:767-777

16. Kovacs Burns K, Nicolucci A, Holt Rl et al (2013) Diabetes Attitudes, Wishes and Needs second study (DAWN2): cross-national benchmarking indicators for family members living with people with diabetes. Diabet Med 30:778-788

17. Holt RI, Nicolucci A, Kovacs Burns K et al (2013) Diabetes Attitudes, Wishes and Needs second study (DAWN2): cross-national comparisons on barriers and resources for optimal care-healthcare professional perspective. Diabet Med 30:789-798

18. McGuire BE, Morrison TG, Hermanns N et al (2010) Short-form measures of diabetes-related emotional distress: the Problem Areas in Diabetes Scale (PAID)-5 and PAID-1. Diabetologia 53:66-69

19. Brähler E, Mühlan $H$, Albani C, Schmidt S (2007) Teststatistsiche Prüfung und Normierung der deutschen Version des EUROHIS-QOL Lebensqualitäts-Index und des WHO 5 Wohlbefindens-Index. Diagnostica 53:83-96

20. Greiner W, Claes C, Busschbach JJ, Schulenburg JM von der (2005) Validating the EQ-5D with time trade off for the German population. Eur J Health Econ 6:124-130

21. Toobert DJ, Hampson SE, Glasgow RE (2000) The summary of diabetes self-care activities measure: results from 7 studies and a revised scale. Diabetes Care 23:943-950

22. Anderson RM, Fitzgerald JT, Gruppen LD et al (2003) The Diabetes Empowerment Scale-Short Form (DES-SF). Diabetes Care 26:1641-1642

23. Schunk M, Reitmeir P, Schipf S et al (2012) Healthrelated quality of life in subjects with and without Type 2 diabetes: pooled analysis of five population-based surveys in Germany. Diabet Med 29:646-653

24. Hermanns N, Kulzer B, Ehrmann D et al (2013) The effect of a diabetes education programme (PRIMAS) for people with type 1 diabetes: results of a randomized trial. Diabetes Res Clin Pract 102:149157

25. Kulzer B, Hermanns N, Reinecker H, Haak T (2007) Effects of self-management training in Type 2 diabetes: a randomized, prospective trial. Diabetic Medicine 24:415-423

26. Hermanns N, Kulzer B, Maier B et al (2012) The effect of an education programme (MEDIAS2 ICT) involving intensive insulin treatment for people with type 2 diabetes. Patient Educ Couns 86:226-232

27. Bergis-Jurgan N, Ehrmann D, HaakT et al (2014) Ambulante Schulung bei Typ-2-Diabetes. Diabetologe 10:293-299

28. Wens J, Vermeire E, Hearnshaw $\mathrm{H}$ et al (2008) Educational interventions aiming at improving adherence to treatment recommendations in type 2 diabetes. A sub-analysis of a systematic review of randomised controlled trials. Diabetes Res Clin Pract 79(3):377-388

29. Clifford S, Barber N, Elliott R et al (2006) Patientcentred advice is effective in improving adherence to medicines. Pharm World Sci 28:165-170 